\title{
Investigation of aminoglycoside resistance inducing conditions and a putative AmrAB-OprM efflux system in Burkholderia vietnamiensis
}

\author{
Agatha N Jassem ${ }^{1,3^{*}}$, Connor M Forbes ${ }^{2,3}$ and David P Speert ${ }^{1,2,3}$
}

\begin{abstract}
Background: Burkholderia cepacia complex (BCC) bacteria are highly virulent, typically multidrug-resistant, opportunistic pathogens in cystic fibrosis (CF) patients and other immunocompromised individuals. B. vietnamiensis is more often susceptible to aminoglycosides than other BCC species, and strains acquire aminoglycoside resistance during chronic CF infection and under tobramycin and azithromycin exposure in vitro, apparently from gain of antimicrobial efflux as determined through pump inhibition. The aims of the present study were to determine if oxidative stress could also induce aminoglycoside resistance and provide further observations in support of a role for antimicrobial efflux in aminoglycoside resistance in B. vietnamiensis.

Findings: Here we identified hydrogen peroxide as an additional aminoglycoside resistance inducing agent in B. vietnamiensis. After antibiotic and hydrogen peroxide exposure, isolates accumulated significantly less $\left[{ }^{3} \mathrm{H}\right]$ gentamicin than the susceptible isolate from which they were derived. Strains that acquired aminoglycoside resistance during infection and after exposure to tobramycin or azithromycin overexpressed a putative resistancenodulation-division (RND) transporter gene, $a m r B$. Missense mutations in the repressor of $a m r B, a m r R$, were identified in isolates that acquired resistance during infection, and not in those generated in vitro.

Conclusions: These data identify oxidative stress as an inducer of aminoglycoside resistance in $B$. vietnamiensis and further suggest that active efflux via a RND efflux system impairs aminoglycoside accumulation in clinical $B$. vietnamiensis strains that have acquired aminoglycoside resistance, and in those exposed to tobramycin and azithromycin, but not hydrogen peroxide, in vitro. Furthermore, the repressor AmrR is likely just one regulator of the putative AmrAB-OprM efflux system in B. vietnamiensis.
\end{abstract}

Keywords: Burkholderia vietnamiensis, Aminoglycoside, Azithromycin, Hydrogen peroxide, Efflux, AmrB, AmrR

\section{Findings}

Members of the Burkholderia cepacia complex (BCC) can cause severe respiratory infections in individuals with cystic fibrosis (CF) [1]. Furthermore, many strains are highly and intrinsically resistant to various antimicrobials, including aminoglycosides [2], ribosome-targeting antibiotics important in the treatment of CF respiratory disease [3].

\footnotetext{
* Correspondence: agatha.jassem@gmail.com

'Department of Pathology and Laboratory Medicine, University of British Columbia, 950 W 28th Avenue, Vancouver, British Columbia V5Z 4H4, Canada ${ }^{3}$ Centre for Understanding and Preventing Infection in Children, Child \& Family Research Institute, University of British Columbia, 950 W 28th Avenue, Vancouver, British Columbia V5Z 4H4, Canada

Full list of author information is available at the end of the article
}

B. cenocepacia studies suggest that resistance-nodulationdivision (RND) efflux systems are involved in BCC resistance to aminoglycosides [4-6]. The MexXY-OprM RND pump is the predominant determinant of aminoglycoside resistance in CF isolates of Pseudomonas aeruginosa [7], and aminoglycoside susceptibility in B. pseudomallei results from loss of AmrAB-OprA [8]. At subinhibitory concentrations, ribosome-targeting antibiotics and oxidative stress induce mex $X Y$ expression $[9,10]$. $\operatorname{mex} X Y$ is under the control of the MexZ repressor [11], and mexZ mutations are common in pan-aminoglycoside resistant isolates [12].

We previously reported that B. vietnamiensis isolates are often aminoglycoside-susceptible and strains acquire resistance during chronic $\mathrm{CF}$ infection and under tobramycin 
and azithromycin pressure in vitro [13]. Decreased access of aminoglycosides to their target resulted from apparent gain of antimicrobial efflux via a RND pump, the latter determined with an inhibitor [13].

\section{B. vietnamiensis develops aminoglycoside resistance under hydrogen peroxide pressure in vitro}

Aminoglycoside resistance can be induced in susceptible $\mathrm{CF}$ isolates of $B$. vietnamiensis following serial exposure to tobramycin (Table 1: C8395TE, D0072TE) or a single exposure to subinhibitory concentrations of azithromycin [13]. To characterize resistance inducing antimicrobial pressures further, after serial passage in cation-adjusted Mueller-Hinton broth (CAMHB) containing azithromycin, meropenem, ceftazidime, and co-trimoxazole at doubling concentrations as described previously [13] the drug susceptibility of C8395 was evaluated. Triplicate minimum inhibitory concentrations (MICs) were determined using broth microdilution methods [14], and their stability confirmed after 20 passages on antibiotic-free media. $P$. aeruginosa and non-Enterobacteriaceae breakpoints were used in the absence of $B$. cepacia breakpoints. Only serial exposure of C8395 to azithromycin resulted in notable ( $\geq 4$ fold) increases in aminoglycoside MICs (Table 1). By previously described methods [9], but with selective agar containing tobramycin at 2.5 times the MIC, serial exposure of C8395 to hydrogen peroxide at half the MIC resulted in a 16-fold stable increase in aminoglycoside MIC for C8395PE (Table 1). Other acquired resistance was also observed: after passage with all antimicrobials the MICs of the respective agents against C8395 increased greatly (Table 1: C8395AE, C8395ME, C8395CE, and C8395SE), and some other cross-resistance, most notably between the $\beta$-lactams antibiotics, was also seen.

Hydrogen peroxide is, therefore, an additional inducer of aminoglycoside resistance in B. vietnamiensis in vitro, a particularly important finding because CF airways are rich in reactive oxygen species [15]. Moreover, B. vietnamiensis can acquire resistance after exposure to other antimicrobials used in treating BCC-infected CF patients, namely meropenem, ceftazidime, and co-trimoxazole [16].

The aminoglycoside-resistant derived isolates C8395TE and C8395PE accumulated 2.65 and 3.50 times less $\left[{ }^{3} \mathrm{H}\right]$ gentamicin than $\mathrm{C} 8395$, respectively $(P=0.0118$, one-way ANOVA) (data not shown). Accumulation was determined in triplicate in Luria-Bertani (LB) medium as previously used to show the late, aminoglycoside-resistant isolate D0774 accumulates less gentamicin than C8395 [13]. There were no significant differences in the $\mathrm{CFU} / \mathrm{ml}$ between $\mathrm{C} 8395$ and the comparison isolates at starting time (data not shown). Decreased access of aminoglycosides to their intracellular target is, therefore, responsible for the observed in vitro antibiotic and oxidative stress-induced resistance.

\section{Analysis of putative efflux system genes in clinical and} in vitro stress exposed $B$. vietnamiensis isolates

Of the 11 putative RND transporters that the sequenced environmental B. vietnamiensis isolate G4 (accession

Table 1 Antimicrobial susceptibilities of B. vietnamiensis after serial exposure to antibiotics or hydrogen peroxide

\begin{tabular}{|c|c|c|c|c|c|c|c|c|c|}
\hline \multirow[t]{2}{*}{ Isolate $^{a}$} & \multicolumn{9}{|c|}{ MIC $(\mu \mathrm{g} / \mathrm{ml})^{b}$} \\
\hline & AMK & GEN & KAN & TOB & AZM & MEM & CAZ & SXT & CIP \\
\hline \multicolumn{10}{|l|}{ Clinical CF } \\
\hline C8395 (3/11/1998, Bv1) & 2 & 4 & 2 & 2 & 32 & 1 & 4 & $2 / 10$ & 1 \\
\hline D0774 (25/7/2003, Bv1) & $>128$ & 128 & 128 & 128 & $>2048$ & 128 & 128 & $64 / 320$ & $>32$ \\
\hline D0072 (15/03/2002, Bv3) & 2 & 4 & 1 & 2 & 32 & 0.5 & 2 & $2 / 10$ & 1 \\
\hline D2910 (31/03/2008, Bv3) & 128 & 32 & 64 & 32 & $>32$ & 2 & 4 & $1 / 5$ & 16 \\
\hline \multicolumn{10}{|l|}{ In vitro exposed } \\
\hline C8395TE (TOB) & $>128$ & $>128$ & 128 & $>128$ & 64 & 1 & 4 & $4 / 20$ & 4 \\
\hline C8395AE (AZM) & 32 & 16 & 16 & 16 & 2048 & 2 & 16 & $8 / 40$ & 4 \\
\hline C8395ME (MEM) & 16 & 8 & 8 & 8 & 32 & 16 & 64 & $4 / 20$ & 16 \\
\hline C8395CE (CAZ) & 8 & 8 & 4 & 4 & 32 & 8 & 16 & $2 / 10$ & 16 \\
\hline C8395SE (SXT) & 8 & 8 & 2 & 2 & 32 & 0.5 & 4 & $>64 / 320$ & 8 \\
\hline C8395PE (peroxide) & 32 & 64 & 32 & 32 & 32 & 4 & 16 & $4 / 20$ & 4 \\
\hline C8395PC (control) & 8 & 8 & 4 & 8 & 32 & 1 & 4 & $1 / 5$ & 1 \\
\hline D0072TE (TOB) & 32 & 32 & 16 & 16 & $>32$ & 1 & 2 & $2 / 10$ & 1 \\
\hline
\end{tabular}

${ }^{a}$ Patient identification numbers and bacterial isolation dates are noted in brackets. Abbreviations: TE, TOB exposed; $A E$, AZM exposed; ME, MEM exposed; CE, CAZ exposed; SE, SXT exposed; PE, hydrogen peroxide exposed; PC, passage control.

${ }^{b}$ Aminoglycoside and azithromycin MICs for C8395 and D0774, and tobramycin and azithromycin MICs for D0072, D2910, C8395TE, and D0072TE were previously published to some extent [13] and are shown here for comparison. MICs represent susceptibility after 3 passages on antibiotic-free media. Abbreviations: AMK, amikacin; GEN, gentamicin; KAN, kanamycin; TOB, tobramycin; AZM, azithromycin; MEM, meropenem; CAZ, ceftazidime; SXT, co-trimoxazole; CIP, ciprofloxacin. 
NC_009256.1) contains (determined as previously [17]), following sequence alignment only Bcep1808_1575 showed high identity, $71 \%, 85 \%$, and $92 \%$, with the characterized transporters MexY (accession NC_008463.1) and AmrB (accession NC_007434.1), and their homologue BCAL1675 in B. cenocepacia (accession NC_011000.1), respectively. These transporters are part of an operon also encoding a repressor, membrane fusion protein, and outer membrane channel [7]. PCR product analysis revealed that B. vietnamiensis clinical isolates C8395, D0774, D0072, and D2910 contained these efflux system genes in the same order (data not shown).

To evaluate the expression of RND pump genes in $B$. vietnamiensis, triplicate overnight cultures were diluted 1:100 into CAMHB, LB medium, or synthetic cystic fibrosis sputum medium (SCFM) [18] with or without tobramycin and ceftazidime at half MIC or azithromycin, meropenem, and co-trimoxazole at a quarter $\mathrm{MIC}$, and grown to an optical density at $600 \mathrm{~nm}\left(\mathrm{OD}_{600}\right)$ of 0.5 or 0.8. RNA was extracted using an RNeasy Plus Mini kit (Qiagen, Toronto, Canada), and treated with RNase-free DNase (Promega, San Luis Obispo, USA). Reverse transcription was performed using SuperScript II Reverse Transcriptase (Invitrogen, Carlsbad, USA) according to the manufacturer's protocol. Amplification of the resultant DNA was quantified in a 7300 Real-Time PCR System (Applied Biosystems, Carlsbad, USA) in the presence of SybrGreen (Invitrogen), with primers for Bcep1808_1575 (5'-CCGAACGACATCTACTTCAAGGTCGG-3', 5'-ATC CTTCGCGACTTCGACGATCAG-3'), Bcep1808_1573 (putative repressor gene) (5'-TGCAGATCCTGCGGATCACGAAA-3', 5'TTCGAGCAACGACACCAGATAGACG-3'), and $16 S$ (for normalization) (5'-CACGCTTTACGCCCAG TAATTCCG-3', 5'-CCGGAAGAATAAGCACCGGCTAAC3 ). Denaturation occurred at $95^{\circ} \mathrm{C}$ for 10 minutes, followed by $40-50$ cycles of 15 seconds at $95^{\circ} \mathrm{C}$ and 1 minute at $60^{\circ} \mathrm{C}$.

The late, aminoglycoside-resistant isolate D0774 expressed 11.4-, 9.6-, and 8.0-fold more Bcep1808_1575, herein " $a m r B$ ", than the early, aminoglycoside-susceptible isolate C8395, at $\mathrm{OD}_{600}$ of 0.8 in CAMHB, LB medium, and SCFM, respectively $(P<0.01)$ (Figure $1 \mathrm{~A})$. $a m r B$ expression between D0774 and C8395 was, however, 2.7 times less at $\mathrm{OD}_{600}$ of 0.5 vs $0.8(P<0.001)$ (Figure $1 \mathrm{~A}$ ), owing to D0774 expressing less $a m r B$ earlier $(P<0.01)$ (data not shown). Compared with C8395, D0774 also expressed 3.6- and 3.2-fold more Bcep1808_1573, herein " $a m r R$ ", at $\mathrm{OD}_{600}$ of 0.8 in CAMHB and SCFM, respectively $(P<0.01)$ (Figure $1 \mathrm{~A})$. In another set of sequential isolates, the late, aminoglycoside-resistant isolate D2910 also overexpressed $a m r B$ (by 5.3-fold) and $a m r R$ (by 2.4-fold) compared with the early, aminoglycosidesusceptible D0072 at $\mathrm{OD}_{600}$ of 0.8 in CAMHB (data not shown).
After a single exposure of C8395 to subinhibitory concentrations of test antimicrobials in CAMHB, $a m r B$ expression did not change (data not shown). After exposure of C8395 to serially doubling concentrations of tobramycin or azithromycin but not to other antibiotics or hydrogen peroxide (i.e. in C8395TE and C8395AE), expression of $a m r B$ increased 9.9- and 8.6-fold, respectively $(P<0.01)$ (Figure $1 \mathrm{~B}$, data not shown). Compared with C8395, C8395TE also expressed 14.2-fold less $\operatorname{amrR}(\mathrm{P}<0.01)$ (Figure 1B).

Active efflux via a RND efflux system is, therefore, probably involved in the decreased drug accumulation observed in B. vietnamiensis strains that acquired aminoglycoside resistance during infection and after exposure to tobramycin and azithromycin in vitro, owing to the correlation between resistance and $a m r B$ expression. Other aminoglycoside resistance determinants exist, since exposure to hydrogen peroxide did not induce $a m r B$ expression, and future tests with minimal medium would demonstrate that absoluteness of these observations. As proposed previously [19], $a m r B$ upregulation only in response to ribosome-targeting agents suggests it is a response to this interaction, not to antibiotics per se. Moreover, $a m r B$ overexpression was not sufficient to cause resistance to non-aminoglycoside antibiotics, supporting the notion that they are not substrates for the putative AmrAB-OprA efflux system [13]. Lastly, there was no association between $a m r R$ expression and aminoglycoside resistance or $a m r B$ expression, as is also true for mex $Z$ [20].

To determine if mutations in amrR were responsible for the observed overexpression of $a m r B$ in B. vietnamiensis, sequences of the putative repressor were examined. DNA isolation, PCR using Phusion High-Fidelity DNA Polymerase (New England Biolabs, Ipswich, USA) with specific primers (5'-TTCAAAGAGGTGTGGGCAGGA-3', 5'-CCGAAACCCGTGTTGTTCATC-3'), and product analysis by agarose gel electrophoresis were done using standard protocols [21]. PCR products were purified with a Wizard SV Gel and PCR Clean-Up System (Promega) and cloned into One Shot TOP10 E. coli cells with a Zero Blunt TOPO PCR Cloning Kit (Invitrogen). Plasmid DNA was isolated using a QIAprep Miniprep Kit (Qiagen), and M13 primers amplified $a m r R$. Resultant products were sequenced at the UBC Centre for Molecular Medicine and Therapeutics.

C8395 and D0774 amrR differed from that of G4 by two silent mutations (data not shown). The late, aminoglycoside-resistant isolate D0774 also contained a substitution at position $425(\mathrm{~T} \rightarrow \mathrm{C})$, that at residue 142 of the protein, in the suggested ligand binding alpha helix region [22], replaces a leucine with a proline. D0072 and D2910 amrR sequences also differed: at position 156, or amino acid residue 52 amid the predicted DNA and 
C-terminal ligand binding domains [22], there was a $\sim 2000$ bp insertion in the late, aminoglycoside-resistant D2910. Only silent mutations were observed in amrR among C8395, C8395TE, C8395AE, C8395PE, and C8395PC (data not shown).

The $a m r R$ mutations identified likely influenced the expression of the putative $B$. vietnamiensis amrB transporter gene. The change in D0774 AmrR may indirectly affect DNA binding to the transcription factor [22], while the large insertion within D2910 amrR likely inactivates the repressor altogether. As per the in vitro derived isolate findings, aminoglycoside-resistant $P$. aeruginosa isolates overexpressing mex $X Y$ without mutations in mex $Z$ also exist $[23,24]$.

In conclusion, in B. vietnamiensis, oxidative stress can induce aminoglycoside resistance, while active efflux via the putative AmrAB-OprM efflux system is likely involved in clinical and in vitro antimicrobial-induced

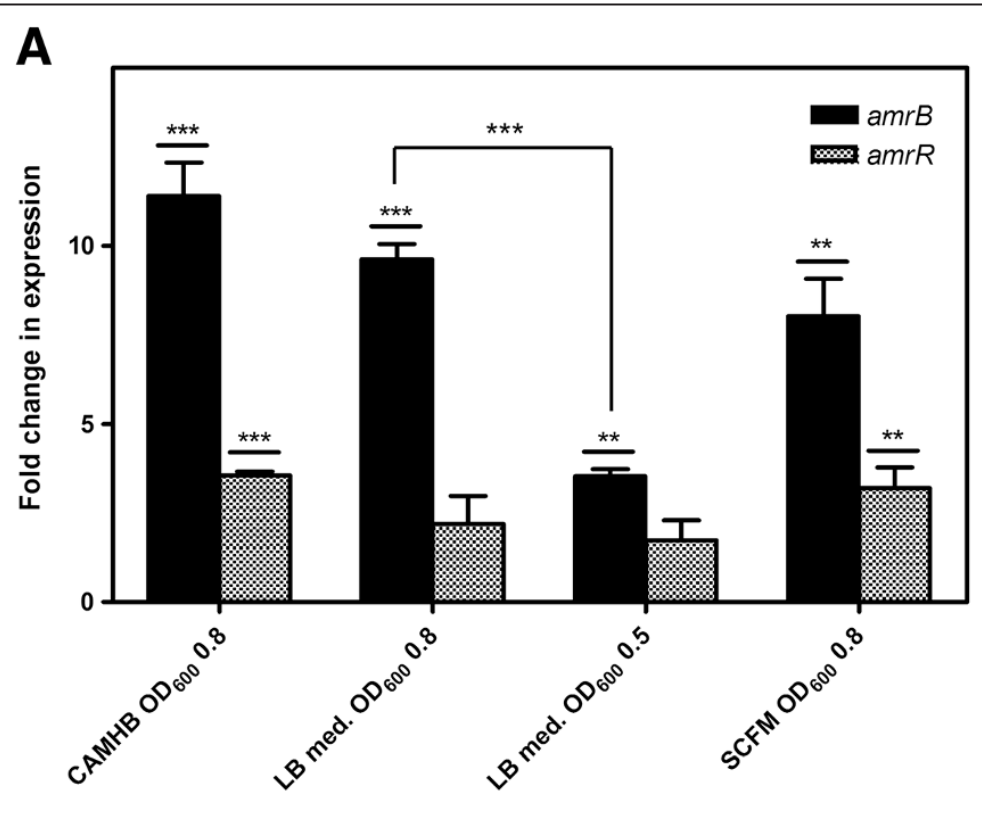

B

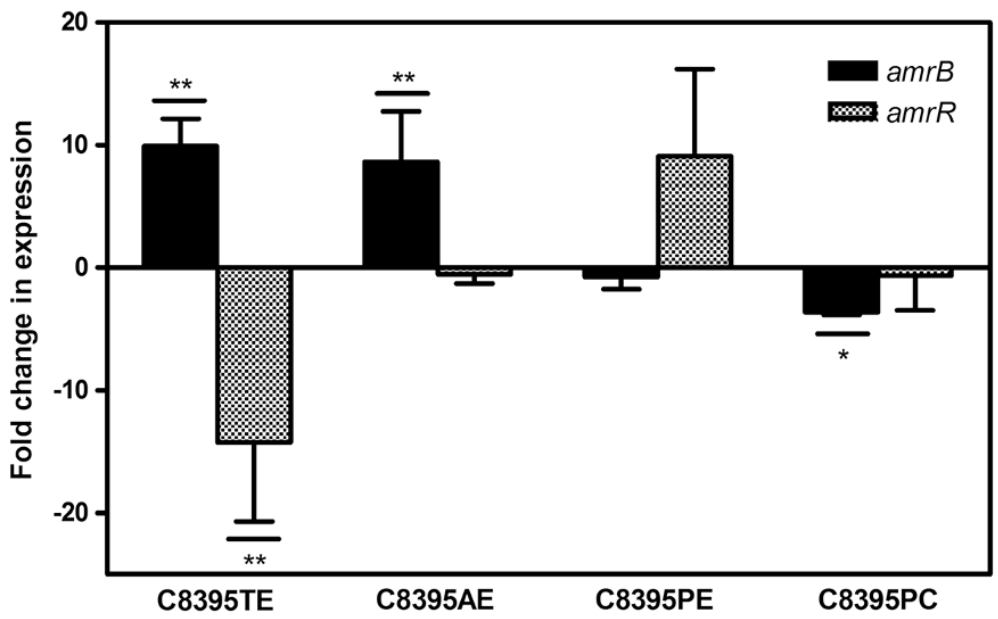

Figure 1 Expression of the putative RND efflux system genes $a m r B$ and $a m r R$ in clinical $C F$ and in vitro antibiotic or hydrogen peroxide exposed $\mathbf{B}$. vietnamiensis isolates. Expression was determined by real-time reverse transcription PCR and compared (A) between the early, aminoglycoside-susceptible isolate C8395, and the late, aminoglycoside-resistant D0774, in various types of media and stages of growth, and (B) between C8395 before and after its exposure to various antimicrobials, peroxide, or passage alone in CAMHB. The averages of three technical repeats were taken for each biological replicate. Fold change means were calculated by comparing the mean expression in C8395 to each biological replicate of (A) D0774 or (B) condition. Data points represent the averages of three biological replicates \pm standard errors. (A) ${ }^{* *}, P<0.01$; ${ }^{* * *} P<0.001$ by unpaired Student's t-test. (B) *, $P<0.05$; ${ }^{* *}, P<0.01$ by Dunnett's Multiple Comparison Test after one-way ANOVA. Abbreviations: amrB, Bcep 1808_1575; amrR, Bcep 1808_1573; RND, resistance-nodulation-division; CAMHB, cation-adjusted Mueller-Hinton broth; LB, Luria-Bertani medium; SCFM, synthetic cystic fibrosis sputum medium; $\mathrm{OD}_{600}$, optical density at $600 \mathrm{~nm}$. 
aminoglycoside resistance. Such elucidation of resistance inducing conditions and resistance factors may improve therapeutic regimens against infection with this species. Additional mechanisms of aminoglycoside resistance should be investigated next. The contribution of resistance determinants to aminoglycoside inefficacy may explain the observed varied degrees of resistance.

\section{Availability of supporting data}

The data supporting the results of this study is included within the article.

\section{Abbreviations}

BCC: Burkholderia cepacia complex; CF: Cystic fibrosis; RND: Resistancenodulation-division; CAMHB: Cation-adjusted Mueller-Hinton broth; MIC: Minimum inhibitory concentration; LB: Luria-Bertani; SCFM: Synthetic cystic fibrosis sputum medium; $\mathrm{OD}_{600}$ : Optical density at $600 \mathrm{~nm}$.

\section{Competing interests}

The authors declare that they have no competing interests.

\section{Authors' contributions}

ANJ designed the study, performed and analyzed experiments not done by CMF, and wrote the manuscript. CMF serially exposed C8395 to hydrogen peroxide and performed most of the susceptibility and expression tests. DPS participated in the design and coordination of the study and critically reviewed the manuscript. All authors read and approved the final manuscript.

\section{Authors' information}

Part of this work was presented at the American Association for the Advancement of Science Annual Meeting, Vancouver, BC, Canada, 16 to 20 February 2012

\section{Acknowledgements}

Support was provided by Cystic Fibrosis Canada (D.P.S. grant; A.N.J., C.M.F studentships) and the Child \& Family Research Institute (C.M.F. studentship). We thank Trevor Hird for technical assistance.

\section{Author details}

${ }^{1}$ Department of Pathology and Laboratory Medicine, University of British Columbia, 950 W 28th Avenue, Vancouver, British Columbia V5Z 4H4, Canada. ${ }^{2}$ Department of Pediatrics, University of British Columbia, $950 \mathrm{~W}$ 28th Avenue, Vancouver, British Columbia V5Z 4H4, Canada. ${ }^{3}$ Centre for Understanding and Preventing Infection in Children, Child \& Family Research Institute, University of British Columbia, 950 W 28th Avenue, Vancouver, British Columbia V5Z 4H4, Canada.

Received: 30 October 2013 Accepted: 1 January 2014

Published: 6 January 2014

\section{References}

1. Mahenthiralingam E, Urban TA, Goldberg JB: The multifarious, multireplicon Burkholderia cepacia complex. Nat Rev Microbiol 2005, 3(2):144-156.

2. Nzula S, Vandamme P, Govan JRW: Influence of taxonomic status on the in vitro antimicrobial susceptibility of the Burkholderia cepacia complex. J Antimicrob Chemother 2002, 50(2):265-269.

3. O'Sullivan BP, Flume P: The clinical approach to lung disease in patients with cystic fibrosis. Semin Respir Crit Care Med 2009, 30(5):505-513.

4. Bazzini $S$, et al: Deciphering the role of RND efflux transporters in Burkholderia cenocepacia. PLoS One 2011, 6(4):e18902.

5. Dubarry N, et al: Improved electrotransformation and decreased antibiotic resistance of the cystic fibrosis pathogen Burkholderia cenocepacia strain J2315. App/ Environ Microbiol 2010, 76(4):1095-1102.

6. Hamad MA, Skeldon AM, Valvano MA: Construction of aminoglycosidesensitive Burkholderia cenocepacia strains for studying intracellular bacteria by the gentamicin protection assay. Appl Environ Microbiol 2010, 76:3170-3176.

7. Poole K: Efflux-mediated antimicrobial resistance. J Antimicrob Chemother 2005, 56(1):20-51.

8. Trunck $L A$, et al: Molecular basis of rare aminoglycoside susceptibility and pathogenesis of Burkholderia pseudomallei clinical isolates from Thailand. PLoS Negl Trop Dis 2009, 3(9):e519.

9. Fraud S, Poole $\mathrm{K}$ : Oxidative stress induction of the MexXY multidrug efflux genes and promotion of aminoglycoside resistance development in Pseudomonas aeruginosa. Antimicrob Agents Chemother 2011, 55(3):1068-1074.

10. Jeannot K, et al: Induction of the MexXY efflux pump in Pseudomonas aeruginosa is dependent on drug-ribosome interaction. J Bacteriol 2005, 187(15):5341-5346.

11. Matsuo $Y$, et al: MexZ-mediated regulation of mexXY multidrug efflux pump expression in Pseudomonas aeruginosa by binding on the mexZmexX intergenic DNA. FEMS Microbiol Lett 2004, 238(1):23-28.

12. Poole K: Pseudomonas aeruginosa: resistance to the max. Front Microbiol 2011, 2:65.

13. Jassem AN, et al: In vitro susceptibility of Burkholderia vietnamiensis to aminoglycosides. Antimicrob Agents Chemother 2011, 55(5):2256-2264

14. CLSI: Methods for dilution antimicrobial susceptibility tests for bacteria that grow aerobically. 9th edition. Wayne, PA: Clinical and Laboratory Standards Institute; 2012. Approved standard M07-A9.

15. Rottner M, Freyssinet JM, Martinez MC: Mechanisms of the noxious inflammatory cycle in cystic fibrosis. Respir Res 2009, 10:23.

16. Avgeri SG, et al: Therapeutic options for Burkholderia cepacia infections beyond co-trimoxazole: a systematic review of the clinical evidence. Int J Antimicrob Agents 2009, 33(5):394-404.

17. Guglierame $P$, et al: Efflux pump genes of the resistance-nodulationdivision family in Burkholderia cenocepacia genome. BMC Microbio/ 2006 6:66.

18. Palmer KL, Aye LM, Whiteley M: Nutritional cues control Pseudomonas aeruginosa multicellular behavior in cystic fibrosis sputum. J Bacteriol 2007, 189(22):8079-8087.

19. Poole K: Bacterial Multidrug Efflux Pumps Serve Other Functions. Microbe 2008, 3:179-185.

20. Yamamoto $M$, et al: Role of MexZ and PA5471 in transcriptional regulation of mexXY in Pseudomonas aeruginosa. Microbiology 2009, 155(Pt 10):3312-3321.

21. Sambrook J, Russell DW: Molecular cloning: a laboratory manual. 3rd edition. Cold Spring Harbor, N.Y: Cold Spring Harbor Laboratory Press; 2001.

22. Alguel $Y$, et al: Crystal structure of MexZ, a key repressor responsible for antibiotic resistance in Pseudomonas aeruginosa. J Struct Biol 2010, 172(3):305-310.

23. Islam S, Jalal S, Wretlind B: Expression of the MexXY efflux pump in amikacin-resistant isolates of Pseudomonas aeruginosa. Clin Microbiol Infect 2004, 10(10):877-883.

24. Sobel ML, McKay GA, Poole K: Contribution of the MexXY multidrug transporter to aminoglycoside resistance in Pseudomonas aeruginosa clinical isolates. Antimicrob Agents Chemother 2003, 47(10):3202-3207.

doi:10.1186/1476-0711-13-2

Cite this article as: Jassem et al.: Investigation of aminoglycoside resistance inducing conditions and a putative AmrAB-OprM efflux system in Burkholderia vietnamiensis. Annals of Clinical Microbiology and Antimicrobials 2014 13:2. 